\title{
ATMOSPHERIC ASCENT GUIDANCE FOR ROCKET-POWERED LAUNCH VEHICLES
}

\author{
Greg A. Dukeman* \\ NASA Marshall Space Flight Center \\ Huntsville, AL 35812
}

\section{Nomenclature}

\begin{abstract}
$\underline{\text { Abstract }}$
An advanced ascent guidance algorithm for rocketpowered launch vehicles is developed. The algorithm cyclically solves the calculus-of-variations two-point boundary-value problem starting at vertical rise completion through main engine cutoff, taking into account atmospheric effects. This is different from traditional ascent guidance algorithms which operate in a simple open-loop mode until the high dynamic pressure portion of the trajectory is over, at which time guidance operates under the assumption of negligible aerodynamic acceleration (i.e., vacuum dynamics). Judicious approximations are made to reduce the order and complexity of the state/costate system. Multiple shooting is shown to be a very effective numerical technique for this application. In particular, just one intermediate shooting point, in addition to the initial shooting point, is sufficient to significantly reduce sensitivity to the guessed initial costates. An abort to downrange landing site formulation of the algorithm is presented. Results comparing guided launch vehicle trajectories with POST open-loop trajectories, for both sub-orbital cutoff conditions and orbit insertion conditions, are given verifying the basic formulation of the algorithm.
\end{abstract}

POST

TAEM

$A$

$C_{A}$

$C_{N}$

g

$H$

$\mathrm{h}$

$M$

$m$

$N$

$q$

$q \alpha$

$q \beta$

$R$

$\mathbf{r}$

$r_{x}, r_{y}, r_{z}$

$S$

$T$

$\mathrm{t}_{\text {Cutoff }}$

V

$\mathrm{V}_{\mathrm{x}}, \mathrm{V}_{\mathrm{y}}, \mathrm{v}_{\mathrm{z}}$

$\mathbf{X}$

$\mathbf{x}_{\mathbf{b}}, \mathbf{y}_{\mathbf{b}}, \mathbf{z}_{\mathbf{b}}$

$\alpha$

$\beta$

$\gamma$

$\delta$

* Guidance and Navigation Specialist, Vehicle

Flight Mechanics Group
Acronyms

Program to Optimize Simulated Trajectories

Terminal Area Energy Management

Symbols

aerodynamic axial force magnitude

coefficient of axial force

coefficient of normal force

gravity acceleration vector

Hamiltonian function

altitude

Mach number

vehicle mass

aerodynamic normal force magnitude

dynamic pressure

product of dynamic pressure and angle of attack

product of dynamic pressure and angle of sideslip

great circle range

vehicle position vector

$\mathrm{x}, \mathrm{y}$, and $\mathrm{z}$ components of position

aerodynamic reference area

thrust magnitude

predicted cutoff time from guidance

vehicle velocity vector

$\mathrm{x}, \mathrm{y}$, and $\mathrm{z}$ components of velocity

column vector containing position and velocity vectors

$\mathrm{x}-, \mathrm{y}-$, and $\mathrm{z}$-body axes unit vectors

vehicle angle of attack

vehicle angle of sideslip

vehicle flight path angle

angle between velocity costate vector and $\mathbf{x}_{\mathbf{b}}$ vector

angle between position vector and velocity costate vector

angle between Earth-relative velocity vector and velocity costate vector 


\begin{tabular}{|c|c|}
\hline & \\
\hline$\Delta T$ & actual thrust minus vacuum thrust \\
\hline$\lambda$ & $\begin{array}{l}\text { column vector containing position } \\
\text { and velocity costates }\end{array}$ \\
\hline$\lambda_{\mathrm{r}}$ & position costate \\
\hline$\lambda_{r y}, \lambda_{r z}$ & components of position vector costate \\
\hline$\lambda_{\mathrm{v}}$ & velocity costate \\
\hline$\sigma_{\text {dir }}$ & $\begin{array}{l}\text { variable set to }+1 \text { or }-1 \text {, specifying } \\
\text { heads-up or heads-down flight, resp. }\end{array}$ \\
\hline$\omega_{0}$ & $\begin{array}{l}\text { parameter associated with invocation } \\
\text { of linear gravity field assumption }\end{array}$ \\
\hline & $\underline{\text { Subscripts }}$ \\
\hline I, $I$ & $\begin{array}{l}\text { vector or quantity relative to the } \\
\text { inertial frame }\end{array}$ \\
\hline $\mathbf{r}, r$ & $\begin{array}{l}\text { vector or quantity relative to the } \\
\text { Earth-fixed frame }\end{array}$ \\
\hline 0 & initial \\
\hline
\end{tabular}

\section{Introduction}

NASA, through a program known as the Space Launch Initiative $^{1}$ (also, Second Generation Reusable Launch Vehicle program), has set for itself the goals of significantly increasing the safety and reliability of the second generation of launch vehicles by two orders of magnitude (to loss of crew in 1 in 10,000 flights), while reducing the launch costs by an order of magnitude (to $\$ 1,000$ /pound payload). In order for the flight mechanics discipline to contribute to these goals, it is important that guidance and control algorithms be highly robust and adaptive to in-flight failures such as partial engine loss.

Traditional ascent guidance algorithms have been relatively simple. The common methodology is to operate in 'open-loop' mode during the (early) high dynamic pressure portion of flight and then, based on a pre-determined time or event, switch to a closed-loop vacuum guidance scheme which operates on the premise that aerodynamic forces can be neglected. The open-loop mode typically makes use of pre-loaded tables of Euler attitude commands versus time or speed $^{2,3}$. The closed-loop logic is based on explicit formulas and simplified dynamics that result in a semianalytical solution for the optimal steering angles. This partitioning of the flight into distinct phases was necessary primarily due to computer throughput and memory limitations as well as unavailability of advanced algorithms that took into consideration aerodynamic forces. Introduction of aerodynamic forces into the problem formulation makes the problem much more sensitive and computationally-intensive. Simple, well-understood formulas don't exist as they do in the vacuum case, making the complete liftoff-toburnout optimization problem difficult to solve reliably in real-time. However, as computer power continues to increase, it will be feasible to use more sophisticated algorithms capable of increasing the reliability and safety of the next generation of launch vehicles.

Some of the earliest research into extending the capabilities of ascent guidance algorithms so that they would be effective just after liftoff was done by Brown, et. al. ${ }^{4}$. In Ref. 4 a linearized aerodynamics model was used to obtain the optimal control (thrust direction) from the optimality condition in closed form. Curve fits for the lift and drag coefficients and atmospheric density, pressure and speed of sound were used in the guidance formulation to reduce the computational burden imposed by aerodynamics modeling. The conventional shooting method combined with a homotopy procedure was used to solve the two-point boundary-value problem. The easily obtained vacuum solution was first computed, from which a homotopy procedure was used to re-introduce increments of the atmospheric effects. Despite the use of homotopy to reduce sensitivity to the initial costate guesses, reliable convergence was not always attained. Kelly develops a similar algorithm formulation in Ref. 5 with similar convergence difficulties reported, despite attempted homotopy procedures, due to inclusion of atmospheric terms. Bradt,et. al. ${ }^{6}$, use a formulation similar to that of Ref. 4 and add a penalty function to reduce bending moment loads. Cramer, et. al. ${ }^{7}$, use a nonlinear programming approach to guidance and take advantage of measured day-of-launch winds in the guidance to provide load relief. Hanson, et. al. develop and test several atmospheric ascent guidance options in Ref. 8. Leung and Calise use a perturbation approach in Ref. 9. Calise and Melamed $^{10}$ use a hybrid collocation approach and demonstrate reliable convergence in dispersed guided trajectory simulations. In Ref. 11, Gath and Calise extend previous work to normal force and angle of attack path constraints and optimization of burn-coast-burn sequences.

The rest of this paper is organized as follows. The next section describes the ascent guidance problem, followed by the formulation of a robust atmospheric ascent guidance formulation suitable for nominal flight. Next, an abort guidance formulation is presented. Numerical results are then given using the guidance formulations. The paper is ended with conclusions and recommendations.

\section{$\underline{\text { Nominal Ascent Guidance Formulation }}$}

\section{$\underline{\text { Trajectory Optimization Problem }}$}

The equations of motion for a thrusting rocket in atmospheric flight are: 


$$
\begin{aligned}
\dot{\mathbf{r}} & =\mathbf{v} \\
\dot{\mathbf{v}} & =\mathbf{g}+\frac{(T-A) \mathbf{x}_{\mathbf{b}}-N \mathbf{z}_{\mathbf{b}}}{m}
\end{aligned}
$$

where the thrust magnitude, $T$, and the axial and normal forces, $A$ and $N$ are given by:

$$
\begin{aligned}
& T=T_{v a c}+\Delta T(h) \\
& A=q S C_{A} \quad N=q S C_{N}
\end{aligned}
$$

Some notes on guidance modeling follow. We've assumed that all the thrust is aligned along the $\mathrm{x}$-body axis, $\mathbf{x}_{\mathbf{b}}$. The velocity vector, $\mathbf{v}$, can be taken as the Earth-relative velocity vector or inertial velocity vector depending on the context. For high-speed flight (typically occurring 'outside' the atmosphere), inertial velocity is used whereas for low-speed flight and suborbital missions, it is sufficient to let the velocity in question be Earth-relative velocity. Note that we've assumed the force along the y-body axis is small and hence we ignore it in the equations of motion and consequently in the optimization (but not in the guided simulations). In the proceeding developments, the position and velocity vectors will be expressed in the guidance coordinate frame illustrated in Figure 1. The guidance coordinate frame is an Earth-centered, righthanded, inertial coordinate system with the $\mathrm{x}$-axis aligned with the local vertical and the $\mathrm{z}$-axis aligned along the expected downrange direction. It is redefined each guidance cycle with the vehicle's current latitude, longitude and an azimuth angle which approximates the downrange direction of travel. This is a convenient frame to work in because, for example, the initial position vector expressed in the guidance frame has $\mathrm{y}$ - and $\mathrm{z}$-components equal to zero and the $\mathrm{y}$ components of position and velocity are typically near zero.

The aerodynamic coefficients are modeled using least squares polynomial coefficients interpolated with cubic spline functions of Mach number, a common technique for reduction of aerodynamics coefficient data in trajectory optimization. The thrust difference term, $\Delta T$, due to the effects of the atmosphere on thrust, can in general be represented by a cubic spline function. The density, $\rho$, is represented by a least squares curve fit of the standard atmosphere and matches the latter to within 5 percent up to $70 \mathrm{~km}$. Similarly, a least squares $3^{\text {rd }}$-order polynomial fit is used to accurately model the speed of sound, $a$.

At the time of engine cutoff, $t_{\text {Cutoff }}, k$ terminal state constraints, nonlinear functions of the states, are imposed:

$$
\begin{aligned}
& \psi_{i}=\psi_{i}\left(\mathbf{r}\left(t_{\text {Cutoff }}\right), \mathbf{v}\left(t_{\text {Cutoff }}\right)\right)=0, \\
& i=1, \ldots, k, \quad k \leq 6
\end{aligned}
$$

Examples of terminal constraints include final position magnitude, flight path angle, semi-major axis, argument of perigee, inclination and longitude of the ascending node.

In general, path constraints of the form:

$$
S(\mathbf{x}, \mathbf{u}, t) \leq 0
$$

are imposed where $\mathbf{x}$ is the state and $\mathbf{u}$ is the control. Examples include maximum axial and normal acceleration, maximum normal force, minimum throttle level, angle of attack, angle of sideslip, and $\min / \max$ values of $q \alpha, q \beta$ and maximum dynamic pressure. The axial force and minimum throttle constraints are fairly straightforward to handle. Gath and Calise considered angle of attack and normal force constraints in Ref. 11 using the mathematical rigor contained in Ref. 12, section 3.10. Constraints on $q \alpha$ and $q \beta$ can be handled in an analogous way because they too are functions of the state and control variables. The maximum dynamic pressure constraint, however, is a state variable constraint. It is well-known that state variable inequality constraints are difficult to treat using optimal control theory. One can also easily conceive of alternative, less rigorous methods of constraining dynamic pressure that may be just as effective and more appropriate for onboard guidance, e.g., Corvin ${ }^{13}$ uses a feedback control law which provides maximum dynamic pressure control via throttle modulation.

We consider now the question of where to point the z-body axis, $\mathbf{z}_{\mathbf{b}}$, which is a function of the control $\mathbf{x}_{\mathbf{b}}$ but is not fully specified given $\mathbf{x}_{\mathbf{b}}$. This decision is a function of how we want the vehicle to fly. We can choose to construct $\mathbf{z}_{\mathbf{b}}$ so that the vehicle flies at zero angle of sideslip:

$$
\mathbf{z}_{\mathbf{b}} \| \mathbf{x}_{\mathbf{b}} \times\left(\mathbf{x}_{\mathbf{b}} \times \mathbf{v}\right)
$$

or so the vehicle flies a zero degree ("heads-up) or 180 degree ("heads-down") bank angle trajectory:

$$
\mathbf{z}_{\mathbf{b}} \| \mathbf{x}_{\mathbf{b}} \times\left(\mathbf{x}_{\mathbf{b}} \times \mathbf{r}_{\mathbf{0}}\right)
$$

The zero-sideslip option requires a non-zero roll angle and possibly excessive roll maneuvering. It is likely that future launch vehicles will have limited roll control authority. The heads-up/heads-down option will inherently result in larger angles of sideslip but this can be attenuated fairly easily, if need be, by imposing 
a sideslip path constraint. In the proceeding, we adopt the heads-up/heads-down option.

The optimization problem can be stated as follows. Determine the $\mathrm{x}$-body axis history, $\mathbf{x}_{\mathbf{b}}(\mathrm{t})$, that maximizes the final vehicle mass (equivalent to minimizing fuel usage or minimizing flight time) subject to the equations of motion (1), the terminal constraints (3) and the path constraints (4)

\section{$\underline{\text { Costate Differential Equations }}$}

We note that the atmospheric portion of flight occurs over a very small ground track, enabling the use of the flat-Earth approximations ${ }^{14}$ :

$$
\begin{gathered}
h \cong r_{x}-r_{E} \\
\mathbf{g} \cong\left[\begin{array}{lll}
g_{0} & 0 & 0
\end{array}\right]^{T}
\end{gathered}
$$

With these, the state equations become (with explicit state dependencies called out):

$$
\begin{aligned}
& \dot{r}_{x}=v_{x} \quad \dot{r}_{y}=v_{y} \quad \dot{r}_{z}=v_{z} \\
& \dot{v}_{x}=-g_{0}+\frac{\left(T\left(r_{x}\right)-A\left(r_{x}, \mathbf{v}\right)\right) x_{b x_{x}}-N\left(r_{x}, \mathbf{v}\right) z_{b x_{x}}}{m} \\
& \dot{v}_{y}=\frac{\left(T\left(r_{x}\right)-A\left(r_{x}, \mathbf{v}\right)\right) x_{b y_{x}}-N\left(r_{x}, \mathbf{v}\right) z_{b y}}{m} \\
& \dot{v}_{z}=\frac{\left(T\left(r_{x}\right)-A\left(r_{x}, \mathbf{v}\right)\right) x_{b z}-N\left(r_{x}, \mathbf{v}\right) z_{b z}}{m}
\end{aligned}
$$

The costate equations then are given by:

$$
\begin{aligned}
& \dot{\lambda}_{r_{y}}=\dot{\lambda}_{r_{z}}=0 \\
& \dot{\lambda}_{r x}=-\frac{\partial H}{\partial r_{x}}=\frac{-\left(T_{h}-A_{h}\right) \boldsymbol{\lambda}_{\mathbf{v}} \cdot \mathbf{x}_{\mathbf{b}}+N_{h} \boldsymbol{\lambda}_{\mathbf{v}} \cdot \mathbf{z}_{\mathbf{b}}}{m} \\
& \dot{\lambda}_{v x}=-\frac{\partial H}{\partial v_{x}}=-\lambda_{r x}+\frac{A_{v x} \boldsymbol{\lambda}_{\mathbf{v}} \cdot \mathbf{x}_{\mathbf{b}}+N_{v x} \boldsymbol{\lambda}_{\mathbf{v}} \cdot \mathbf{z}_{\mathbf{b}}}{m} \\
& \dot{\lambda}_{v y}=-\frac{\partial H}{\partial v_{y}}=-\lambda_{r y}+\frac{A_{v y} \boldsymbol{\lambda}_{\mathbf{v}} \cdot \mathbf{x}_{\mathbf{b}}+N_{v y} \boldsymbol{\lambda}_{\mathbf{v}} \cdot \mathbf{z}_{\mathbf{b}}}{m} \\
& \dot{\lambda}_{v z}=-\frac{\partial H}{\partial v_{z}}=-\lambda_{r z}+\frac{A_{v z} \boldsymbol{\lambda}_{\mathbf{v}} \cdot \mathbf{x}_{\mathbf{b}}+N_{v z} \boldsymbol{\lambda}_{\mathbf{v}} \cdot \mathbf{z}_{\mathbf{b}}}{m}
\end{aligned}
$$

where the subscripts $h, v x, v y$, and $v z$ denote partial differentiation with respect to those variables. No known analytic solutions for the atmospheric state/costate system exist so we resort to a second-order Runge-Kutta numerical integration scheme to propagate the state/costate system. Ten integration steps are sufficient to obtain a good guidance solution.
In the next subsection, we apply the maximum principle to obtain the optimal control for atmospheric flight.

Optimality Condition

Applying the maximum principle to the Hamiltonian results in the optimization sub-problem:

$$
\max _{\mathbf{x}_{\mathbf{b}}}\left\{\boldsymbol{\lambda}_{v} \cdot\left(\frac{(T-A) \mathbf{x}_{\mathbf{b}}-N \mathbf{z}_{\mathbf{b}}}{m}\right)\right\}
$$

Note that the optimal control, $\mathbf{x}_{\mathbf{b}}{ }^{\mathbf{0}}$ (and hence, optimal z-body axis, $\mathbf{z}_{\mathbf{b}}{ }^{\mathbf{}}$ ) lies in the plane defined by the (initial) position and velocity-costate vectors. Thus, with reference to Figure 2 (after Gath and Calise ${ }^{11}$ ), the optimization sub-problem (10) can be written simply as

$$
\max _{\delta}\{(T-A) \cos (\delta)+N \sin (\delta)\}
$$

Note that we need to be able to evaluate $\alpha$ in terms of $\delta$ so that we can evaluate $A$ and $N$ in the previous equation. From Figure 2, it is clear that $\alpha$ is some constant, $\alpha_{0}$, (that is, constant with respect to $\delta$ ) minus $\delta$ :

$$
\alpha=\alpha_{0}-\delta
$$

The formula for $\alpha_{0}$ can be derived by setting $\delta$ to zero and solving for $\alpha$ via:

$$
\begin{aligned}
& v_{x_{b}}=\mathbf{v} \cdot \mathbf{x}_{\mathbf{b}}=v \cos \left(\phi_{0}\right) \\
& \mathbf{z}_{\mathbf{b}}=\sigma_{d i r} \frac{\mathbf{x}_{\mathbf{b}} \times\left(\mathbf{x}_{\mathbf{b}} \times \hat{\mathbf{r}}_{\mathbf{0}}\right)}{\sin (\phi)}=\sigma_{d i r} \frac{\cos (\phi) \mathbf{x}_{\mathbf{b}}-\hat{\mathbf{r}}_{\mathbf{0}}}{\sin (\phi)} \\
& v_{z_{b}}=\mathbf{v} \cdot \mathbf{z}_{\mathbf{b}}=\sigma_{d i r} \frac{\left[v \cos (\phi) \cos \left(\phi_{0}\right)-v_{x}\right]}{\sin (\phi)} \\
& \alpha_{0}=\tan ^{-1}\left(\frac{\sigma_{d i r}\left[v \cos (\phi) \cos \left(\phi_{0}\right)-v_{x}\right]}{v \sin (\phi) \cos \left(\phi_{0}\right)}\right)
\end{aligned}
$$

In the preceding, note that $\phi$ and $\phi_{0}$ are simple functions of the state and costate.

The maximization sub-problem can be solved in many ways. One option is to take the derivative with respect to $\delta$, set to zero and use an iterative procedure (e.g., Newton's method) to get the root which corresponds to the optimum $\delta$. This approach was found to be problematic because there are situations when the Hamiltonian (as a function of $\delta$ ) is very flat and Newton's method is very slow to converge. A more direct method is to do a Golden Section search ${ }^{15}$. The Golden Section algorithm is relatively inefficient but the function to be optimized in this case, the Hamiltonian, is fairly inexpensive to evaluate. 
Once the Hamiltonian is maximized, we need to construct the $\mathrm{x}$ - and $\mathrm{z}$-body axes so that we can evaluate the state/costate differential equations. Start by expressing the $\mathrm{x}$-body axis as a linear combination of costate and initial position vector

$$
\mathbf{x}_{\mathbf{b}}=a_{1} \hat{\lambda}_{\mathbf{v}}+b 1 \hat{\mathbf{r}}_{\mathbf{0}}
$$

Dot the preceding with velocity costate and with initial position to get

$$
\begin{aligned}
& \cos (\delta)=\hat{\lambda}_{\mathbf{v}} \cdot \mathbf{x}_{\mathbf{b}}=a_{1}+b_{1} \cos (\phi) \\
& \cos (\phi+\delta)=\hat{\mathbf{r}}_{\mathbf{0}} \cdot \mathbf{x}_{\mathbf{b}}=a_{1} \cos (\phi)+b_{1}
\end{aligned}
$$

Solve the preceding two equations for $a_{1}$ and $b_{1}$ in terms of the angles $\phi$ and $\delta$ :

$$
\begin{aligned}
& a_{1}=\frac{\cos (\delta)-\cos (\phi) \cos (\phi+\delta)}{\sin ^{2}(\phi)} \\
& b_{1}=\frac{\cos (\phi+\delta)-\cos (\phi) \cos (\delta)}{\sin ^{2}(\phi)}
\end{aligned}
$$

The z-body axis is constructed via:

$$
\begin{aligned}
& \mathbf{z}_{\mathbf{b}}=\mathbf{x}_{\mathbf{b}} \times \mathbf{y}_{\mathbf{b}}=\frac{\sigma_{d i r}}{\sin (\phi+\delta)} \mathbf{x}_{\mathbf{b}} \times\left(\mathbf{x}_{\mathbf{b}} \times \hat{\mathbf{r}}_{\mathbf{0}}\right) \\
& =\frac{\sigma_{d i r}}{\sin (\phi+\delta)}\left[\begin{array}{c}
\left(a_{1} \cos (\phi)+b_{1}\right)^{2}-1 \\
a_{1} \lambda_{v_{y}}\left(a_{1} \cos (\phi)+b_{1}\right) \\
a_{1} \lambda_{v_{z}}\left(a_{1} \cos (\phi)+b_{1}\right)
\end{array}\right]
\end{aligned}
$$

\section{$\underline{\text { Transversality Conditions }}$}

The transversality conditions consist of conditions on final costate, referred to in the sequel as 'costate conditions', and on the final Hamiltonian:

$$
\begin{aligned}
& \lambda^{\mathbf{T}}\left(t_{f}\right)=\mathbf{v}^{\mathbf{T}} \boldsymbol{\psi}_{\mathbf{x}} \\
& H\left(t_{f}\right)=1
\end{aligned}
$$

where $v$ is a column-vector of constant Lagrange multipliers, $\psi$ is the column-vector of terminal state constraints and $\psi_{\mathrm{x}}$ is the 'constraint gradients matrix', that is, the $k$ by 6 matrix whose $k$ rows are the gradients (wrt state, $\mathbf{x}$ ) of the terminal state constraints. The costate conditions are equivalent to requiring that the final costate vector be orthogonal to the space spanned by all admissible final state variations $\delta \mathbf{x}$. For a given set of $k$ terminal state constraints, expressions for $6-k$ admissible, or 'transversality vectors', are obtained a priori and the inner products of the final costate with these vectors are iteratively driven to zero (using a modified Newton's method) simultaneously with the terminal state constraints. As an example, whenever argument of perigee is free, the admissible state variation corresponds to a rotation about the angular momentum vector, hence, one of the 6- $k$ required transversality vectors is ${ }^{16}$ :

$$
\delta x=\left[\begin{array}{c}
\mathbf{r} \times \mathbf{h} \\
\mathbf{v} \times \mathbf{h}
\end{array}\right]=\left[\begin{array}{l}
(\mathbf{r} \cdot \mathbf{v}) \mathbf{r}-r^{2} \mathbf{v} \\
v^{2} \mathbf{r}-(\mathbf{r} \cdot \mathbf{v}) \mathbf{v}
\end{array}\right]
$$

Note that, because the costates can be arbitrarily scaled by a positive factor, the end-point condition on the Hamiltonian is equivalent to requiring that the Hamiltonian be positive. This condition is usually satisfied and is indeed satisfied whenever all the terminal state constraints are Keplerian constants (e.g., semi-major axis, eccentricity) and the costate conditions are satisfied. To see this note that for any Keplerian constant, $\psi$

$$
\dot{\psi}=\psi_{\mathbf{x}} \dot{\mathbf{x}}=\psi_{\mathbf{x}}\left[\mathbf{v}^{T},-\mu \frac{\mathbf{r}^{T}}{\|\mathbf{r}\|^{3}}\right]^{T}=0
$$

hence, $\left[\mathbf{v}^{\mathbf{T}}, \mathbf{g}(\mathbf{r})^{\mathrm{T}}\right]^{\mathrm{T}}$ is a transversality vector whenever all the terminal constraints are Keplerian constants (also, whenever true anomaly is free) and thus satisfaction of the costate conditions imply that the 'Keplerian' part of the Hamiltonian (at the final time) is zero. Now, note that the sign of the non-Keplerian part of the Hamiltonian is of definite sign:

$$
\lambda_{\mathbf{v}}^{\mathbf{T}} \frac{T}{m} \hat{\mathbf{u}}=\left\|\boldsymbol{\lambda}_{\mathbf{v}}\right\| \frac{T}{m}>0
$$

Hence, $H\left(t_{f}\right)>0$ and the end-point condition on the Hamiltonian can generally be replaced by the simpler (non-constraining) condition that the final costate magnitude be unity.

\section{$\underline{\text { Vacuum Guidance Formulation }}$}

Whenever dynamic pressure is reasonably small, say, less than $50 \mathrm{psf}$, the vacuum assumption can be invoked. Under the assumption of linear steering, i.e.,:

$$
x_{b}(t)=\frac{\mathbf{A} t+\mathbf{B}}{\|\mathbf{A} t+\mathbf{B}\|}
$$


and constant thrust magnitude or constant thrust acceleration, a closed-form solution exists for the thrust integral requiring no numerical integration or quadrature $^{17}$. The gravity integral is obtained using a predictor-corrector step. Because the gravity vector doesn't change significantly over the ascent trajectory, one step is usually sufficient.

Next, for the costate vacuum solution, we assume a linear central gravity field ${ }^{18-19}$, that is:

$$
\mathbf{g}(\mathbf{r}) \cong-\frac{\mu}{r_{0}^{3}} \mathbf{r} \equiv-\omega_{0}^{2} \mathbf{r}
$$

so that the costate differential equations become:

$$
\left[\begin{array}{l}
\dot{\boldsymbol{\lambda}}_{\mathbf{r}} \\
\dot{\boldsymbol{\lambda}}_{\mathbf{v}}
\end{array}\right]=\left[\begin{array}{cc}
\mathbf{0}_{\mathbf{3}} & \omega_{0}^{2} \mathbf{I}_{\mathbf{3}} \\
-\mathbf{I}_{\mathbf{3}} & 0_{3}
\end{array}\right]\left[\begin{array}{l}
\boldsymbol{\lambda}_{\mathbf{r}} \\
\boldsymbol{\lambda}_{\mathbf{v}}
\end{array}\right]
$$

whose solution is:

$$
\begin{aligned}
& \lambda_{\mathbf{r}}(t)=\cos \left(\omega_{0} t\right) \lambda_{\mathbf{r}}\left(t_{0}\right)+\omega_{0} \sin \left(\omega_{0} t\right) \boldsymbol{\lambda}_{\mathbf{v}}\left(t_{0}\right) \\
& \lambda_{\mathbf{v}}(t)=-\frac{\sin \left(\omega_{0} t\right)}{\omega_{0}} \lambda_{\mathbf{r}}\left(t_{0}\right)+\cos \left(\omega_{0} t\right) \lambda_{\mathbf{v}}\left(t_{0}\right)
\end{aligned}
$$

Typically, for a given mission, a good initial guess for the mission-elapsed time at which dynamic pressure becomes small is available. Up until that 'simulated' time, the atmospheric equations developed earlier are used in the (numerical) propagation of state/costate. At that point, the current propagated state/costate is handed off to the 'vacuum propagator' (after Ref. 8). It is advantageous to do this, that is, only use the atmospheric equations when absolutely necessary, because the atmospheric equations are much more complicated than the analogous vacuum equations. Note that it is not critical that the predicted time of 'atmospheric exit', $t_{E x o}$, be especially accurate. If need be, the predicted dynamic pressure at $t_{E x o}$ can be monitored and $t_{E x o}$ increased if necessary.

\section{$\underline{\text { Numerical Solution Method }}$}

The multiple shooting method ${ }^{20}$ is used to solve the resulting two-point boundary-value problem. The values of the six initial costates, and the engine cutoff time are the free variables that must be iterated upon to null the $k$ terminal state constraints, the $6-k$ costate conditions and the constraint on final costate magnitude. Multiple shooting allows the user to guess state/costate values at more than just the initial point, significantly reducing the well-known sensitivity to the initial costates. In fact, it has been found that insertion of just one additional shooting point (besides the initial shooting point), placed just after peak dynamic pressure, dramatically reduces the sensitivity compared to single shooting.

\section{Abort Guidance Formulation}

The abort guidance formulation is very similar to the nominal guidance formulation. In the case of abort to downrange or abort to launch site, the target values are obtained from an entry profile ${ }^{21}$, i.e., a table of reference altitude, speed, and flight path versus rangeto-HAC 'break points', available from onboard data used (or generated) by the entry guidance function. An additional constraint for aborts to a landing site is that fuel be depleted during the ascent burn so that, e.g., landing gear loads are not exceeded.

A set of terminal constraints for a downrange abort case is given by:

$$
\begin{aligned}
& r-r_{d}=0 \\
& \mathbf{v} \cdot \mathbf{r}-r_{d} v_{d} \sin \left(\gamma_{d}\right)=0 \\
& \mathbf{v} \cdot\left(\left(\mathbf{r} \times \mathbf{r}_{\mathbf{H A C}}\right) \times \mathbf{r}\right)-v_{d} \cos \left(\gamma_{d}\right) r^{2} \sin (\theta)=0 \\
& \mathbf{v} \cdot\left(\mathbf{r} \times \mathbf{r}_{\mathbf{H A C}}\right)=0
\end{aligned}
$$

where the HAC radius vector, $\mathbf{r}_{\mathbf{H A C}}$, is a unit vector directed from the center of the Earth to the heading alignment cone, the desired values (' $d$ ' subscripts) are obtained from the re-entry profile, and the range angle, $\theta$, is the angle subtended by the vehicle position vector and the HAC radius vector. The first constraint fixes the altitude, the second and third fix the vertical and horizontal speeds, resp., and the fourth nulls the vehicle heading error with respect to the HAC. Note that the third ensures that the vehicle is actually headed toward the HAC and not away from it.

For the case of downrange aborts, we want to burn all the propellant so, instead of optimizing fuel usage, we choose to maximize final speed $v$. The transversality conditions are:

$$
\begin{aligned}
& \left.\left(\boldsymbol{\lambda}-\boldsymbol{\varphi}_{\mathbf{x}}\right)\right|_{t=t_{f}} \cdot \boldsymbol{\delta} \mathbf{x}_{\mathbf{i}}=0, \quad i=1,2 \\
& H\left(t_{f}\right)=\boldsymbol{\lambda} \cdot \mathbf{f}=\boldsymbol{\lambda}_{r} \cdot \mathbf{v}-\boldsymbol{\lambda}_{v} \cdot \frac{\mu}{r^{3}} \mathbf{r}=0
\end{aligned}
$$

where we've assumed that we intercept the re-entry profile on a coast arc, $\varphi_{\mathrm{x}}$ is the gradient of the performance index with respect to state, $\mathbf{x}$, and where the 'admissible' state vectors, $\delta \mathbf{x}_{\mathbf{i}}$, are given by:

$$
\delta \mathbf{x}_{1}=\left[\begin{array}{l}
\mathbf{r} \times \mathbf{r}_{\text {HAC }} \\
\mathbf{v} \times \mathbf{r}_{\text {HAC }}
\end{array}\right]
$$


and

$$
\boldsymbol{\delta} \mathbf{x}_{\mathbf{2}}=\left[\begin{array}{c}
\frac{\partial r}{\partial R} \frac{\mathbf{r}}{r}+\frac{r}{R_{e}} \frac{\mathbf{h} \times \mathbf{r}}{|\mathbf{h} \times \mathbf{r}|} \\
\frac{\partial v}{\partial R} \frac{\mathbf{v}}{v}-v \frac{\mathbf{h} \times \mathbf{v}}{|\mathbf{h} \times \mathbf{v}|}\left(\frac{\partial \gamma}{\partial R}-\frac{1}{R_{e}}\right)
\end{array}\right]
$$

The transversality vector $\delta \mathbf{x}_{1}$ reflects the fact that a perturbation of the final position normal to the flight plane combined with a perturbation of the final velocity normal (but opposite in sign) to the flight plane is admissible. The transversality vector $\delta \mathbf{x}_{2}$ is a calculation of the admissible change in final position and velocity given a perturbation of final range (treated as a free variable).

The transversality conditions (27) can be written:

$$
\begin{aligned}
& \lambda \cdot \boldsymbol{\delta} \mathbf{x}_{1}-\varphi_{\mathbf{x}} \cdot \boldsymbol{\delta} \mathbf{x}_{1}=0 \\
& \boldsymbol{\lambda} \cdot \boldsymbol{\delta} \mathbf{x}_{2}-\varphi_{\mathbf{x}} \cdot \boldsymbol{\delta} \mathbf{x}_{2}=0 \\
& H\left(t_{f}\right)=0
\end{aligned}
$$

or, equivalently as:

$$
\begin{aligned}
& \lambda \cdot \boldsymbol{\delta} \mathbf{x}_{\mathbf{1}}-\varphi_{\mathbf{x}} \cdot \boldsymbol{\delta} \mathbf{x}_{\mathbf{1}}=0 \\
& \left(\varphi_{\mathbf{x}} \cdot \boldsymbol{\delta} \mathbf{x}_{1}\right)\left(\lambda \cdot \boldsymbol{\delta} \mathbf{x}_{2}\right)-\left(\boldsymbol{\lambda} \cdot \boldsymbol{\delta} \mathbf{x}_{1}\right)\left(\varphi_{\mathbf{x}} \cdot \boldsymbol{\delta} \mathbf{x}_{2}\right)=0 \quad(31 \mathrm{a}, \mathrm{b}, \mathrm{c}) \\
& H\left(t_{f}\right)=0
\end{aligned}
$$

Suppose all the necessary conditions are satisfied except (31a). Then, (31a) can be solved by scaling the costate vector by an appropriate factor. Note that the arbitrary scaling has no effect on the necessary conditions or on the trajectory. If we enforce $(31 \mathrm{~b})$ instead of both (31a) and (31b), we can retain our logic from the nominal guidance formulation wherein we keep the initial costate vector normalized, $\left|\lambda_{v}\left(\mathrm{t}_{0}\right)\right|=1$ and impose the non-constraining constraint $\left|\lambda_{\mathrm{v}}\left(\mathrm{t}_{\mathrm{f}}\right)\right|=1$. Now, substituting $v$ for $\varphi$, we have:

$$
\varphi_{x}=\left[\begin{array}{llllll}
0 & 0 & 0 & \frac{v_{x}}{v} & \frac{v_{y}}{v} & \frac{v_{z}}{v}
\end{array}\right]
$$

Then, using (31b), (31c), and the second of (32), the transversality conditions reduce to the simple form:

$$
\begin{aligned}
& \boldsymbol{\lambda} \cdot \boldsymbol{\delta} \mathbf{x}_{\mathbf{1}}=0 \Rightarrow \boldsymbol{\lambda}_{\mathbf{r}} \cdot\left(\mathbf{r} \times \mathbf{r}_{\mathbf{H A C}}\right)+\boldsymbol{\lambda}_{\mathbf{v}} \cdot\left(\mathbf{v} \times \mathbf{r}_{\mathbf{H A C}}\right)=0 \\
& H\left(t_{f}\right)=0
\end{aligned}
$$

To sum up the constraints, we have the four final state constraints (26), the transversality conditions (33), and the (non-constraining) constraint $\left|\lambda_{\mathrm{v}}\left(t_{f}\right)\right|=1$. The parameters to be iteratively determined are the six initial costates and the time of flight.

\section{Numerical Results}

The algorithm formulation as described above has been coded in $\mathrm{C}$ and integrated into the high-fidelity MAVERIC/X-33 trajectory simulator which includes 6 degrees of freedom, a day-of-launch winds model, and the GRAM atmosphere model. For sub-orbital missions, the X-33 vehicle model was used. To enable orbital insertion missions, the Isp of the X-33 vehicle was doubled. The end of the atmospheric phase was taken as 140 seconds inside the guidance solution process whereafter the vacuum solution is used. One intermediate shooting point was used. A $q \alpha$ constraint of 500 psf-deg was imposed inside the guidance solution process. For the two orbital missions, an axial acceleration limit of $3.5 \mathrm{~g}$ 's was imposed. As a rough indicator of algorithm efficiency, the 3 degree-offreedom simulations run significantly faster than realtime on a three-year old DEC alpha computer/processor. The initial guess was generated from the vacuum solution although other simpler methods would probably work just as well. Results for three mission simulations are shown here: 1) A 6 degree-of-freedom sub-orbital mission simulation launching from Edwards Air Force Base to Michael Army Air Field in Utah, 2) a 3 degree-of-freedom orbital mission launching from Kennedy Space Center to a 100 by 100 nautical mile circular, 28.5 degree inclination orbit, and 3) a 3 degree-of-freedom orbital mission launching from Kennedy Space Center with simulated 50 percent thrust loss at $90 \mathrm{~s}$ into the mission requiring a downrange abort to a runway in Spain. For the first mission, the X-33 vehicle model ${ }^{3}$ was used and for the remaining two, the X-33 vehicle with increased Isp was used. The guidance in all cases was executed at $1 \mathrm{~Hz}$ with complete updates (re-optimized trajectories) each cycle starting at liftoff, although for the first four seconds, the guidance solution was ignored and the guidance commands were set to execute a vertical rise. In all cases, open-loop reference trajectories (from POST) are available and comparison plots were made to verify acceptable performance of the guidance. In the orbital case, the guided trajectory was very comparable to the open-loop trajectory with the guided trajectory out-performing the POST trajectories by an insignificant 200 pounds fuel.

Figures 3-5 pertain to the sub-orbital mission. Figure 3 compares the ascent altitude and flight path angle histories of the MAVERIC closed-loop and POST open-loop simulations. Although the histories are noticeably different in the middle of the trajectory, 
the end-points are virtually identical. Figure 4 shows the $\mathrm{q} \alpha$ and $\mathrm{q} \beta$ hisories and Figure 5 shows the ground track.

Figures 6-8 pertain to the $100 \mathrm{nmi}$ circular orbit insertion mission. Again, very comparable performance and insertion accuracy are obtained. The ground tracks differ by the geodetic to geocentric correction.

Figures 9-15 pertain to the abort-to-downrange site mission. Figure 9 shows the time and magnitude of the thrust loss. Figure 10 shows the altitude and speed profiles for the entire ascent through entry. The latter phase was flown with closed-loop entry guidance to further verify that the ascent guidance delivered the vehicle to an entry-compatible state. Figure 11 shows the ground track which is compatible with landing in Spain. Figure 12 shows the angle of attack and sideslip. Apparently, the optimal thing to do to adapt to thrust loss is to pitch up. Figure 13 shows $q \alpha$ and $q \beta$ which are both fairly benign. Figure 14 shows the heading error with respect to the HAC. This verifies that the ascent guidance is successful at nulling heading error at main engine cutoff. Figure 15 shows the commanded cutoff time, an output from guidance. The prediction is quasi-constant indicating that the guidance is sufficiently accurately modeling aerodynamics, gravity and thrust.

\section{$\underline{\text { Summary and Conclusions }}$}

This paper describes an ascent guidance algorithm that re-optimizes the entire ascent trajectory each guidance cycle from liftoff to main engine cutoff. High-fidelity guided trajectories compared with POST open-loop trajectories demonstrate that it provides near-optimal performance despite flat-Earth simplifications of the costate equations. The highfidelity trajectory simulator with the guidance cycling at $1 \mathrm{~Hz}$ runs significantly faster than real-time, indicating the algorithm's efficiency. An abort-todownrange site formulation is given along with guided abort trajectory results.

Future work will involve using day-of-launch winds in the guidance solution and incorporating Mach-scheduled angle of attack constraints. Several candidate RLVs are 2-stage vehicles with gimbaled engines, possibly requiring modification of the assumption that all thrust is directed along the x-body axis.

\section{Acknowledgement}

The POST trajectory data generated by Terri Schmitt of the Vehicle Flight Mechanics Group at NASA-Marshall is gratefully acknowledged.

\section{$\underline{\text { References }}$}

${ }^{1}$ Anon., "The Space Launch Initiative: Technology to Pioneer the Space Frontier," NASA Marshall Space Flight Center, Pub. 8-1250, FS-2001-06-122-MSFC, June, 2001.

${ }^{2}$ McHenry, R. L., Brand, T. J., Long, A. D., Cockrell, B. F., and Thibodeau, J. R. III, "Space Shuttle Ascent Guidance, Navigation, and Control", Journal of the Astronautical Sciences, Vol. XXVII, No. 1, pp. 1-38, January-March, 1979.

${ }^{3}$ Hanson, J. M., Coughlin, D. J., Dukeman, G. A., Mulqueen, J. A., McCarter, J. W., "Ascent, Transition, Entry, and Abort Guidance Algorithm Design for the X-33 Vehicle", AIAA Paper 98-4409, 1998.

${ }^{4}$ Brown, K. R., Harrold, E.F., Johnhson, G. W., "Some New Results on Space Shuttle Atmospheric Ascent Optimization", AIAA Paper No. 70-978, 1970.

${ }^{5}$ Kelly, W. D., "Formulation of Aerodynamic Quantities for Minimum Hamiltonian Guidance”, AIAA Paper 92-4380, 1992.

${ }^{6}$ Bradt, J. E., Jessich, M. V., and Hardtla, J. W., "Optimal Guidance for Future Space Applications," AIAA Paper 872401, August 17-29, 1987, Monterey, CA.

${ }^{7}$ Cramer, E. J., Bradt, J. E., Hardtla, J. W., "Launch Flexibility Using NLP Guidance and Remorte Wind Sensing", AIAA Paper 90-3330-CP, 1990.

${ }^{8}$ Hanson, J., Shrader, M., Cruzen, C., "Ascent Guidance Options", AIAA Paper 94-3568-CP, 1994.

${ }^{9}$ Leung, M. S. K., Calise, A. J., "A Hybrid Approach to NearOptimal Launch Vehicle Guidance”, AIAA Paper 92-4304CP, 1992.

${ }^{10}$ Calise, A. J., Melamed, N., and Lee, Seungjae, "Design and Evaluation of a Three-Dimensional Optimal Ascent Guidance Algorithm", Journal of Guidance, Control, and Dynamics, vol. 21, no. 6, pp. 867-875, Nov-Dec 1998.

${ }^{11}$ Gath, P., and Calise, A., "Optimization of Launch Vehicle Ascent Trajectories With Path Constraints and Coast Arcs", AIAA Paper 99-4308, August 1999.

${ }^{12}$ Bryson, Arthur, E., Jr., and Ho, Yu-Chi, "Applied Optimal Control", Hemisphere Publishing Corporation, 1975.

${ }^{13}$ Corvin, M.A., 'Ascent Guidance for a Winged Boost Vehicle', NASA-CR-172083, August 1988.

${ }^{14}$ Vinh, N.X., "Integrals of the Motion for Optimal Trajectories in Atmospheric Flight", AIAA J., 11, (1973), 700-703.

${ }^{15}$ Press, W.H., Teukolsky, S.A., Vetterling, W.T., Flannery, B.P., "Numerical Recipes in C: The Art of Scientific Computing", $2^{\text {nd }}$ ed., Cambridge University Press, 1992.

${ }^{16}$ Teren, Fred, and Spurlock, Omer F., "Optimal Three

Dimensional Launch Vehicle Trajectories with Attitude and

Attitude Rate Constraints", Lewis Research Center, NASA

Technical Note, NASA TN-D-5117, NASA, Washington,

D.C., March 1969.

${ }^{17}$ Burrows, R. R., McDaniel, G. A., “A Method of Trajectory Analysis With Multi-Mission Capability and Guidance Application”, AIAA Paper No. 68-844, August 1968. 
${ }^{18}$ Jezewski, D.J., "Optimal Analytic Multiburn Trajectories," AIAA Journal, Vol. 10, No.5, 1972, pp. 680-685.

${ }^{19}$ Azimov, D.M., Bishop, R.H., "Extremal Rocket Motion with Maximum Thrust in a Linear Central Field," Journal of Spacecraft and Rockets, Vol. 38, No.5, September-October 2001, pp. 765-776.

${ }^{20}$ Stoer, J., and Bulirsch, R., "Introduction to Numerical Analysis", Springer-Verlag, New York, 1980.

${ }^{21}$ Lu, P., Shen, Z., Dukeman, G., Hanson, J., "Entry

Guidance by Trajectory Regulation”, AIAA paper 2000-3958,

Proceedings of Guidance, Navigation and Control

Conference, August 14-17, 2000, Denver, CO.

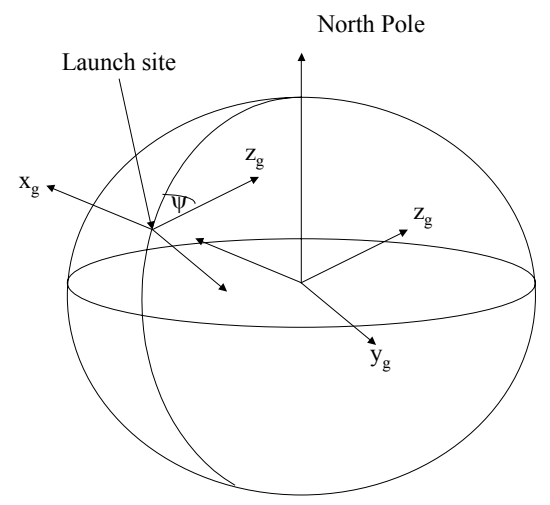

Figure 1: Guidance Reference Frame

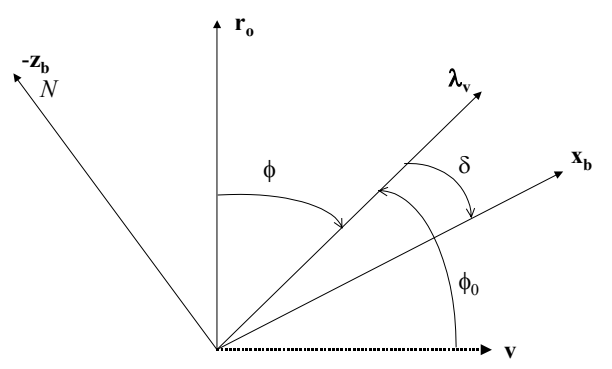

Figure 2: Angles Pertaining To Optimality Condition

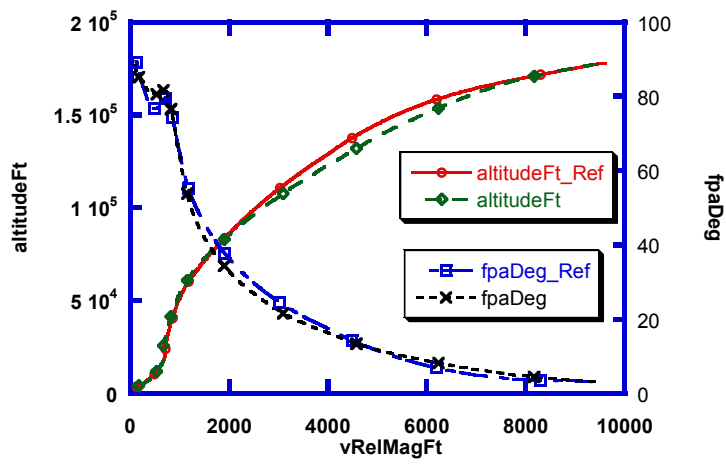

Figure 3: Altitude/flight path, POST/MAVERIC

Comparison, Sub-orbital Mission

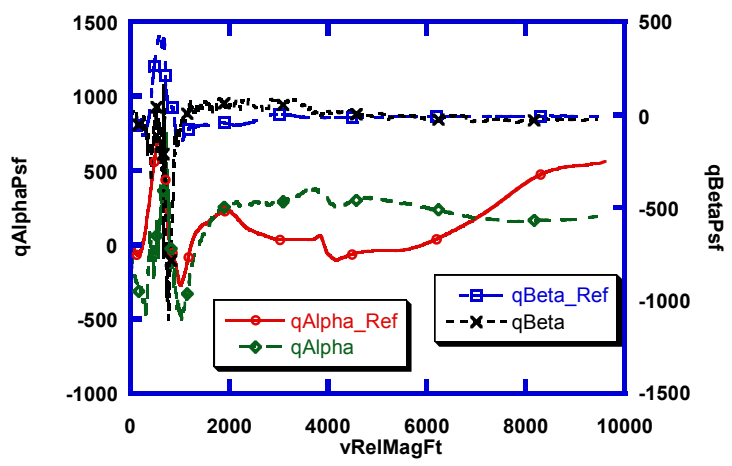

Figure 4: $\mathrm{q}-\alpha, \mathrm{q}-\beta$, POST/MAVERIC Comparison, Sub-orbital Mission 


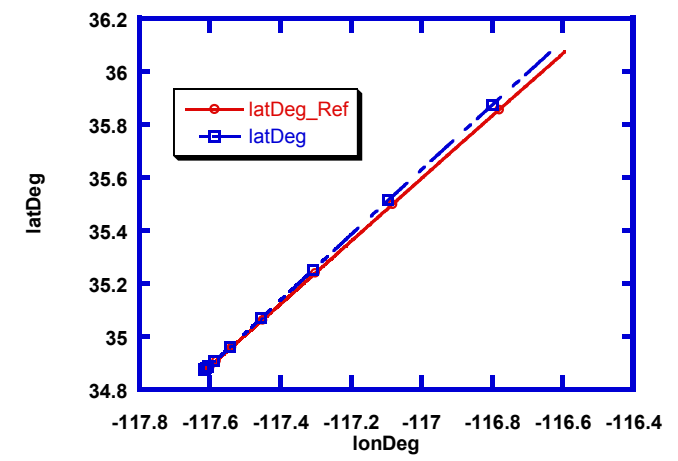

Figure 5: Ground Track, POST/MAVERIC Comparison, Sub-orbital Mission

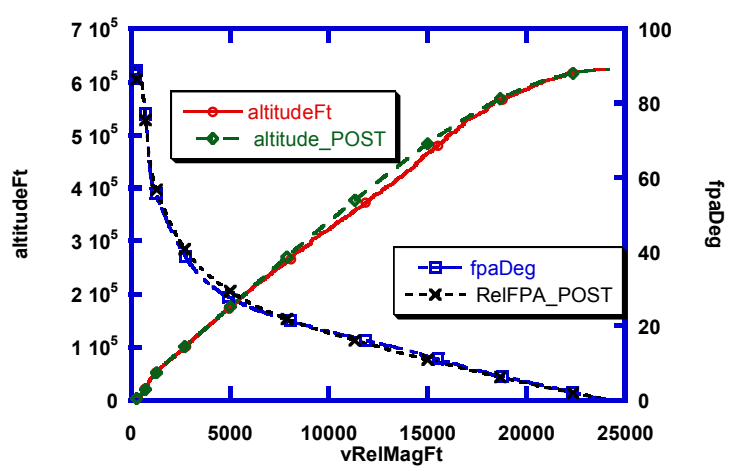

Figure 6: Altitude/flight path, POST/MAVERIC Comparison, Orbital Mission

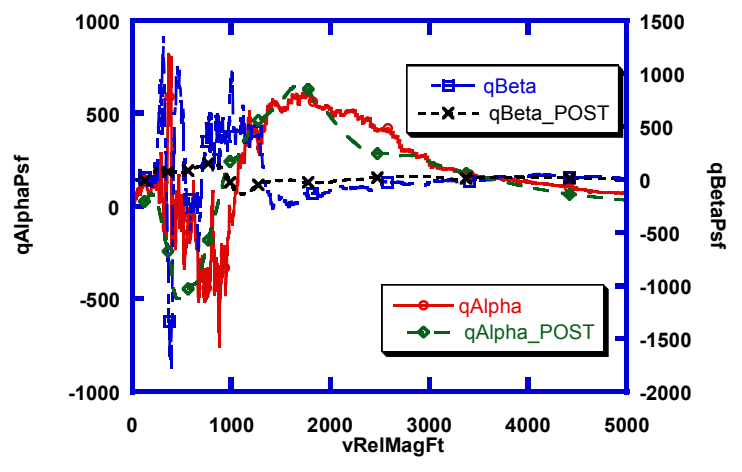

Figure 7: $\mathrm{q}-\alpha, \mathrm{q}-\beta$, POST/MAVERIC Comparison, Orbital Mission

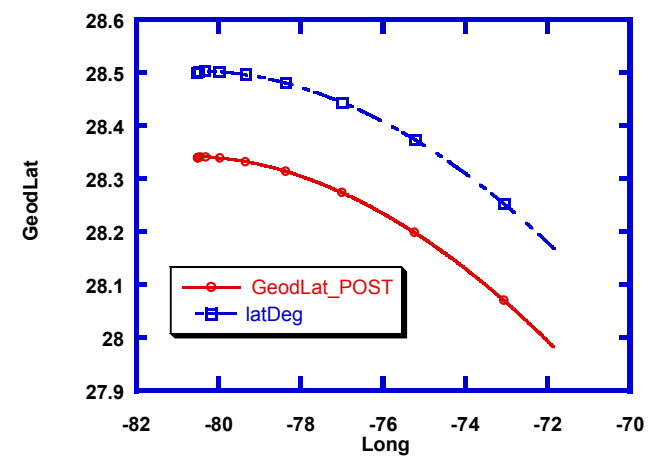

Figure 8: Ground Track, POST/MAVERIC Comparison, Orbital Mission

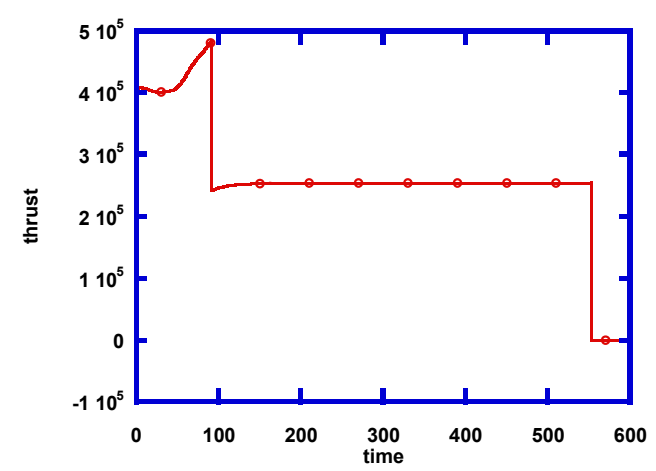

Figure 9. Thrust (lbf), Abort Mission

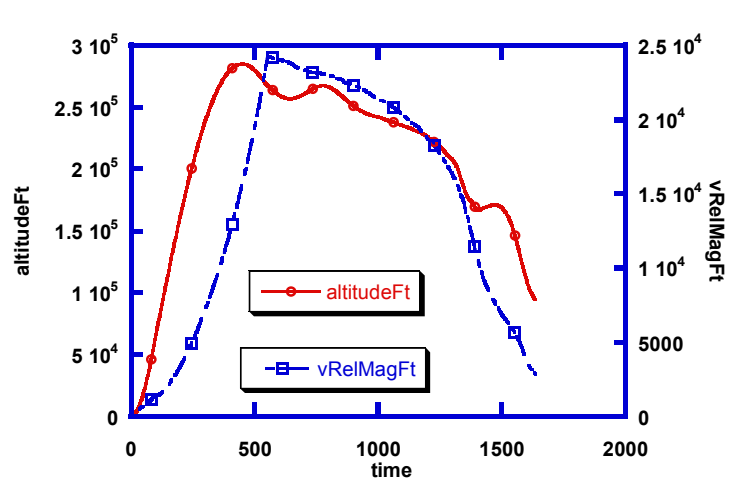

Figure 10: Altitude/Speed, Abort Mission 


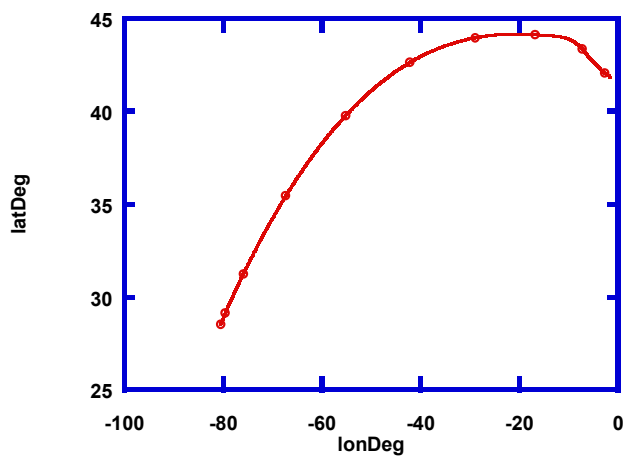

Figure 11: Ground Track, Abort Mission

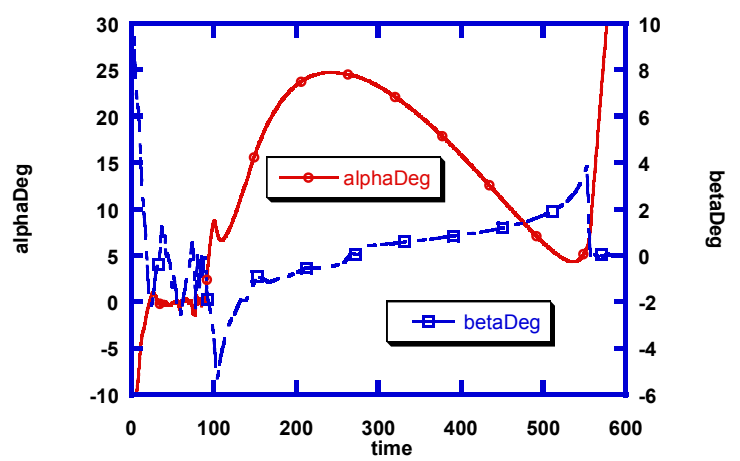

Figure 12: Angle of attack/sideslip, Abort mission

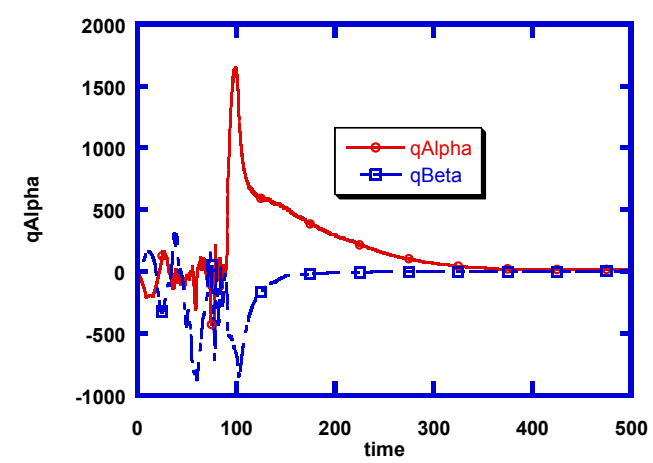

Figure 13: $\mathrm{q} \alpha, \mathrm{q} \beta$, Abort mission

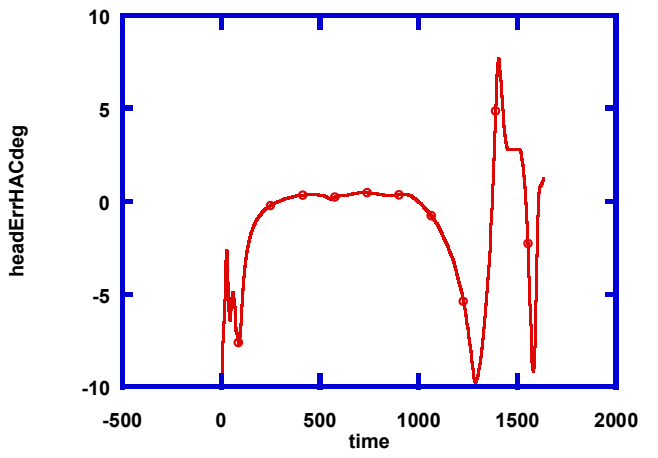

Figure 14: HAC heading error, Abort mission

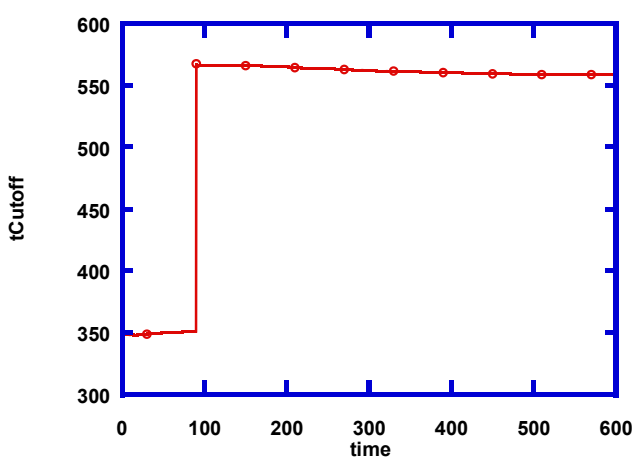

Figure 15: Guidance predicted cutoff time, Abort mission 